\title{
Retraction Note to: Secular trends in major osteoporotic fractures among 50+ adults in Denmark between 1995 and 2010
}

\author{
Shahab Abtahi ${ }^{1,2,3}$. Johanna H. M. Driessen ${ }^{1,2,3,4}$. Peter Vestergaard ${ }^{5,6} \cdot$ Joop van den Bergh ${ }^{4,7,8,9}$. \\ Annelies Boonen ${ }^{2,7}$. Frank de Vries ${ }^{1,2,3}$ • Andrea M. Burden 1,2,3
}

Published online: 8 July 2019

(C) International Osteoporosis Foundation and National Osteoporosis Foundation 2019

\section{Retraction Note to: Archives of Osteoporosis (2018) 13: 91 https://doi.org/10.1007/s11657-018-0503-z}

The publisher has retracted this article [1] from Archives of Osteoporosis in order to publish in Osteoporosis International as originally intended by the authors and the Editors of Osteoporosis International. Springer Nature apologizes to readers and the authors. All authors agree to this retraction.

1. Abtahi S, Driessen JH, Vestergaard P, et al (2018) Arch Osteoporos 13:91. https://doi.org/10.1007/s11657-018-0503-Z

Publisher's note Springer Nature remains neutral with regard to jurisdictional claims in published maps and institutional affiliations.

The online version of the original article can be found at https://doi.org/ $10.1007 / \mathrm{s} 11657-018-0503-\mathrm{z}$

Frank de Vries

frank.de.vries@mumc.nl

1 Department of Clinical Pharmacy and Toxicology, Maastricht University Medical Center+, Maastricht, The Netherlands

2 Care and Public Health Research Institute (CAPHRI), Maastricht, The Netherlands

3 Division of Pharmacoepidemiology and Clinical Pharmacology, Utrecht Institute of Pharmaceutical Sciences,

Utrecht, The Netherlands

4 NUTRIM School for Nutrition and Translational Research in Metabolism, Maastricht University Medical Center+, Maastricht, The Netherlands
5 Department of Clinical Medicine, Aalborg University, Aalborg, Denmark

6 Department of Endocrinology, Aalborg University Hospital, Aalborg, Denmark

7 Department of Internal Medicine, Division of Rheumatology, Maastricht University Medical Center+, Maastricht, The Netherlands

8 Department of Internal Medicine, VieCuri Medical Center, Venlo, The Netherlands

9 Faculty of Medicine and Life Sciences, Hasselt University, Hasselt, Belgium 